\title{
Influence of vacancies on metallic nanotube transport properties
}

\author{
Neophytos Neophytou, ${ }^{a)}$ Shaikh Ahmed, and Gerhard Klimeck \\ School of Electrical and Computer Engineering, Purdue University, West Lafayette, Indiana 47907-1285
}

(Received 26 December 2006; accepted 11 April 2007; published online 4 May 2007)

\begin{abstract}
The electronic behavior of metallic carbon nanotubes under the influence of atomistic vacancy defects present in the channel is theoretically investigated using non-equilibrium Green's function method self-consistently coupled with three-dimensional electrostatics. A nearest neighbor tight binding model based on a single $p_{z}$ orbital is used for the device Hamiltonian. A single vacancy defect in the channel of a small diameter metallic carbon nanotube can decrease its conductance by a factor of 2. More than one vacancy in the channel can further drastically decrease the conductance. Larger diameter nanotubes suffer less from the presence of vacancy defects. (C) 2007 American Institute of Physics. [DOI: 10.1063/1.2736295]
\end{abstract}

Single-walled carbon nanotubes (CNTs) are of great interest for future electronic and other applications due to their excellent electronic and optical properties. Near-ballistic transport with exceptionally high mobilities, is one of their characteristics which makes them attractive for circuit implementations. Transistor structures that demonstrate highperformance operation have been reported. ${ }^{1-4}$ Metallic nanotubes have also been given a large attention for the possibility of their use as interconnects in very large scale integrated circuits ${ }^{5}$ and field emission devices. ${ }^{6,7}$

Significant theoretical work has been done in investigating the performance of perfect CNT devices. It is well known, however, that vacancies arise in graphite at low concentrations during defective growth or as part of the thermal equilibrium concentration. 8,9 They are much more prevalent in irradiated materials and are believed to be the predominant defects on irradiated graphite surfaces and CNTs and stable on a long time scale. ${ }^{10-13}$ The understanding of the performance of structures with defects is therefore important, especially when CNTs are used in high radiation environment, such as space applications. A previous work investigated the performance of vacancy defected semiconducting carbon nanotube field effect transistors (CNTFETs). ${ }^{14}$ This work examines the influence of vacancy defects on the electronic transport in metallic carbon nanotubes and finds a very significant influence on the device behavior.

The model device considered here consists of a $(12,0)$ zigzag CNT, with a $1 \mathrm{~nm}$ diameter (Fig. 1). The source and drain are metallic electrodes of length $5 \mathrm{~nm}$ and radius $2 \mathrm{~nm}$. The surrounding gate oxide is a $4 \mathrm{~nm}$ thick $\mathrm{HfO}_{2}$ high $\kappa$ dielectric material $(\kappa=16)$. The interior of the CNT is vacuum $(\kappa=1)$. The work functions of the source/drain and gate electrodes are assumed to be equal to the work function of the CNT, $\left(\Phi_{S / D}=\Phi_{\text {gate }}=\Phi_{\mathrm{CNT}}\right)$. In this case, the Fermi level of the contacts aligns with the Fermi level of the CNT channel (which is assumed to be at $0 \mathrm{eV}$ ), and the gate tends to keep the CNT intrinsic at zero bias. Our simulator includes a full three-dimensional (3D) treatment of electrostatics, and current and electron density calculations are based on real space non-equilibrium Green's function (NEGF) technique within the nearest neighbor tight binding $\pi$-orbital approximation. ${ }^{15,16}$ To model the vacancy, we set the on-site

${ }^{a)}$ Electronic mail: neophyto@purdue.edu potential to $10^{6} \mathrm{eV}$ at the carbon vacancy site, which ensures that a channel electron is effectively repelled from the location of the vacancy. ${ }^{17}$ In this study we do not include effects of structure relaxation due to the vacancy.

Introduction of a vacancy defect in the channel of the CNT alters both the density of states and the transmission of the CNT near the Fermi level due to the change in bonding between the vacancy and its nearest neighbors and introduces localized states. In Figs. 2(a) and 2(b), the non-selfconsistent simulated density of states $[\operatorname{DOS}(E)]$ and transmission $[T(E)]$ for a $(12,0)$ CNT are shown, respectively, with and without a single vacancy present in the channel. The model device in this case is just a two unit cell channel, with open boundary conditions for the contacts. The density of states around the Fermi level [Fig. 2(a)] rises due to the broken $\pi$ network at the vacancy site. The transmission on the other hand [Fig. 2(b)] decreases close to one quantum unit $\left(q^{2} / h\right.$ per spin, where $q$ is the charge of the electron and $h$ is Plank's constant) in the vicinity of the Fermi level in agreement with Chico et al. ${ }^{18}$ For CNTs with larger diameters, i.e., $(42,0)$ with $4 \mathrm{~nm}$ diameter, similar behavior is observed as in the case of the $(12,0) \mathrm{CNT}$, as shown in Figs. 2(c) and 2(d). In this case, however, the reduction in transmission is more localized near the middle of the energy spectrum of the CNT. The same effect is observed for the increase in the density of states, which is mostly localized in the middle of the energy spectrum. These differences will significantly affect the $I-V$ characteristics of the two devices.

The self-consistent simulated charge and potential energy distribution along the channel of the coaxial $(12,0)$ CNTFET of Fig. 1 are shown in Figs. 3(a) and 3(b). The vacancy is placed in the middle of the channel. Figure 3(a) indicates an accumulation of charge at the vacancy location,

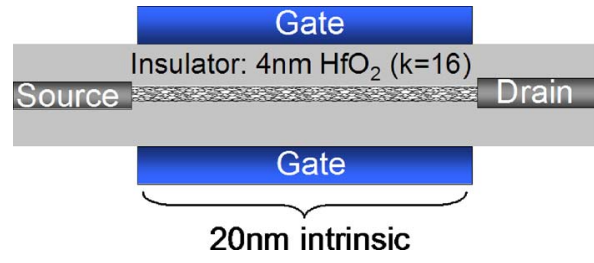

FIG. 1. (Color online) 3D coaxial CNTFET with $20 \mathrm{~nm}$ channel length and metallic source/drain extensions. The channel is a metallic $(12,0)$ zigzag CNT with $1 \mathrm{~nm}$ diameter. The gate oxide is assumed to be a $4 \mathrm{~nm}$ thick $\mathrm{HfO}_{2}$ with dielectric constant $k=16$. 

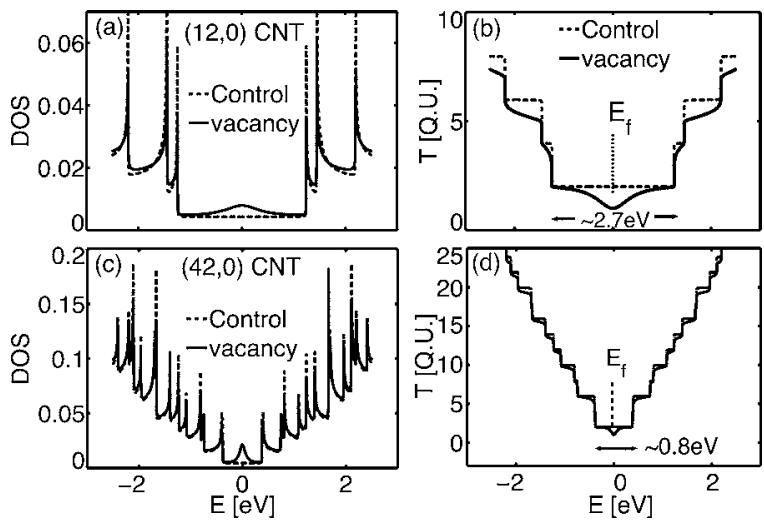

FIG. 2. Density of states $[\mathrm{DOS}(E)]$ and transmission $[T(E)]$ for CNT channels consisting of two unit cells with open boundary conditions. (a) The $\operatorname{DOS}(E)$ for a $(12,0)$ CNT with $\sim 1 \mathrm{~nm}$ diameter for the control (defect-free, dash line) vs vacancy included (solid line). The unit cell consists of 48 atoms. (b) The $T(E)$ of the control $(12,0)$ CNT (dash) vs the defected one (solid). (c) The $\operatorname{DOS}(E)$ for a $(42,0) \mathrm{CNT}$ with $\sim 4 \mathrm{~nm}$ diameter for the control (defect-free, dash), vs vacancy included (solid). The unit cell consists of 168 atoms. (d) The $T(E)$ of the control $(42,0)$ CNT (dash) unit cell vs the defected one (solid).

which is associated with the increased local $\operatorname{DOS}(E)$. The amount of charge increases as the bias on the gate electrode increases, because more of the vacancy induced states are now filled with electrons. This charge accumulation raises the electrostatic energy of the channel locally [Fig. 3(b)]. Under the nonzero gate and drain biases $\left(V_{G}=0.5 \mathrm{~V}\right.$, $V_{D}=0.2 \mathrm{~V}$ ), shown in Figs. 3(c) and 3(d), the local accumulation of charge near the defect site creates a scattering center, and causes changes in the potential profile and the carrier
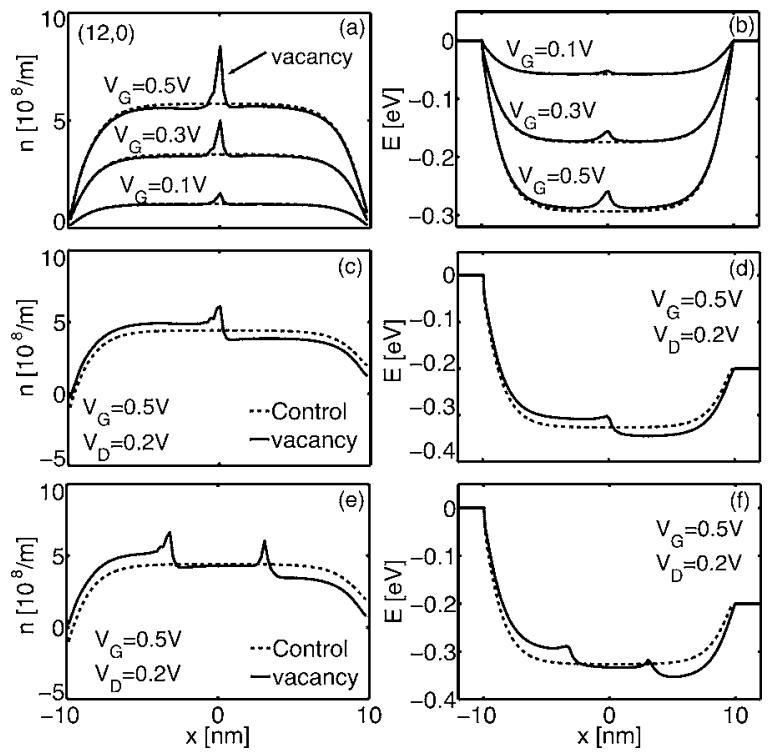

FIG. 3. Effect of the vacancy on the electronic properties of a metallic $(12,0)$ CNTFET. (a) The charge distribution along the channel of the control (dash) vs the vacancy contained (solid) CNTFET for $V_{G}=0.1,0.3$, and $0.5 \mathrm{~V}$, under equilibrium conditions $\left(V_{D}=0 \mathrm{~V}\right)$. (b) The corresponding potential energy profile for the cases described in (a). (c) The charge distribution along the channel of the control and the defected CNTFET for $V_{G}$ $=0.5 \mathrm{~V}$, under nonequilibrium conditions $\left(V_{D}=0.2 \mathrm{~V}\right)$. Large scattering is observed at the vacancy site. Charge is accumulated in the left of the vacancy. (d) The corresponding potential energy profile for the case described in (c). (e) The charge distribution along the channel of the control and the defected with two vacancies CNTFET for $V_{G}=0.5 \mathrm{~V}$, under nonequilibrium conditions $\left(V_{D}=0.2 \mathrm{~V}\right)$. (f) The corresponding potential energy profile for the case described in (e). Downloaded 04 May 2007 to 128.46.212.67. Redistribution subject to AIP license or copyright, see http://apl.aip.org/apl/copyright.jsp
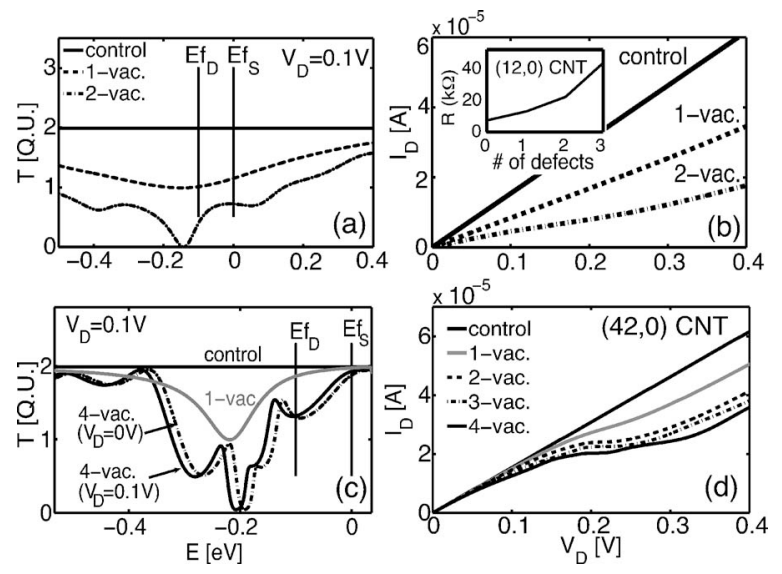

FIG. 4. Effect of the vacancy defects on the drive current capabilities of the CNTFET. (a) The self-consistent transmission coefficient of the $(12,0)$ CNTFET with none (solid), one (dash), and two (dash-dot) vacancies in the channel. The window $E f_{S}-E f_{D}$ indicates the important electron transport energy region. (b) $I_{D}$ vs $V_{D}$ for control vs vacancy defected $(12,0)$ CNTFETs. Control (solid), single vacancy (dash), and two vacancies (dash dot) in the channel. Inset: The resistance of the channel as a function of the number of vacancies. (c) The self-consistent transmission coefficient of the $(42,0)$ CNTFET for the control (solid), single vacancy (solid gray), and four-vacancy device (solid) at $V_{D}=0.1 \mathrm{~V}$. Also, the four-vacancy device at $V_{D}=0.1 \mathrm{~V}$ (dash dot). In all cases, the vacancies are placed in equal distances from each other in the channel, i.e., in the case of the two vacancies, they are placed at $L / 3$ and $2 L / 3$ distances in the channel, where $L$ is the channel length. (d) $I_{D}$ vs $V_{D}$ for control vs vacancy defected $(42,0)$ CNTFETs.

occupancy of the channel in its entire length. There is an accumulation and a depletion of the carrier density $(n)$ to the left and right of the vacancy, respectively, due to carrier reflections from the localized state, as indicated in Fig. 3(c). This causes the potential energy profile to float up (down) at the left (right) sides of the vacancy [Fig. 3(d)]. Introduction of two vacancies in the channel of the device, as shown in Figs. 3(e) and 3(f), has a similar local effect in the places where the vacancy is present. In this case the vacancies are located at $L / 3(\sim 6.7 \mathrm{~nm})$ and $2 L / 3(\sim 13 \mathrm{~nm})$ from the source contact, where $L$ is the length of the channel $(20 \mathrm{~nm})$. The two peaks in the charge distribution indicate the location of the defects, which cause pileup of the charge and the potential energy to the left side of the device and depletion to the right side.

The $I_{D^{-}} V_{D}$ characteristics are computed by selfconsistent NEGF simulations performed in the ballistic limit. The current is calculated in the Landauer formalism as $I=\int T(E)\left(E f_{S}-E f_{D}\right) d E$, where $\left(E f_{S}-E f_{D}\right)$ is the difference between the Fermi levels of the source and the drain electrodes. As indicated in Fig. 4(a), the self-consistent transmission of the control $(12,0)$ CNT device is constant at two quantum units over the entire energy range of interest (within the $E f_{S}-E f_{D}$ window). For a device with a single vacancy, the transmission is reduced to one quantum unit, and for the case of two vacancies in the channel, it reduces even further. Since the reduction happens over a large energy range, shifting the $E f_{S}-E f_{D}$ window (for example, by using different gate biases or different work functions for the gate), will not help in improving the defected devices' transmission probabilities. Figure 4(b) shows the self-consistently computed $I_{D^{-}} V_{D}$ characteristics for a $(12,0) \mathrm{CNT}$ with and without vacancy defects in the channel. Because of the constant transmission of the control CNT around the Fermi level, the me- 
tallic CNT $I_{D}-V_{D}$ characteristics are linear with the slope being the conductance of the channel, as shown in Fig. 4(b) (solid line). A single vacancy defect in the middle of the channel reduces the conductance by a factor of 2 (dashed line). Two and three vacancies are considered, placed in equal distances from one another in the channel. The drive current reduces by almost half every time another defect is introduced in the channel and the corresponding channel resistance increases [inset of Fig. 4(b)]. It is also found that the exact position of the defect does not significantly alter the $I_{D^{-}} V_{D}$ characteristics. Variations in the defect position can cause small shifts and oscillations in the transmission spectrum; however, they cannot affect the overall drive current characteristics significantly.

The control $(42,0)$ CNT, with larger diameter $(\sim 4 \mathrm{~nm})$, has the same transmission as the $(12,0) \mathrm{CNT}$ (two quantum units), and therefore delivers the same amount of current under small drain biases. [The $(42,0) \mathrm{CNT}$ will deliver more current at higher drain biases since other energy bands appear in much lower energies than the $(12,0) \mathrm{CNT}]$. The transmission plots of the control CNT, the CNT with one and four vacancies for the case of $V_{D}=0.1 \mathrm{~V}$, are shown in Fig. 4(c). The transmission curve for the case of the four-vacancy CNT at $V_{D}=0 \mathrm{~V}$ (dotted line) is also shown for comparison to the four-vacancy device at $V_{D}=0.1 \mathrm{~V}$. This shows that the shape of the transmission of a certain channel does not significantly change with $V_{D}$, except from a small shift in its energy spectrum (smaller than the $V_{D}$ window). The transmission curves for the cases of two and three vacancies fall in between these curves; however, for simplicity they are not shown here. The $I_{D^{-}} V_{D}$ characteristics for both control and defected $(42,0)$ CNTs are shown in Fig. 4(d). [Due to numerical convergence issues at high biases for the defected devices, the $I_{D}-V_{D}$ characteristics were extracted for the entire bias range by using the self-consistent transmission coefficient derived at $V_{D}=0.1 \mathrm{~V}$, considering that the shape of the transmission profile does not change significantly with $V_{D}$, as shown in Fig. 4(c)]. At low drain biases, the drive current in all the devices is almost unaffected. The reason is that the reduction in transmission for all the defected devices is more localized within a smaller energy range [compared to the $(12,0)$ CNT case] and initially falls out of the $E f_{S}-E f_{D}$ window of width $0.1 \mathrm{~V}$. This, however, depends on the choice of the initial placement of this energy window (by changing the gate bias or the work functions of the electrodes). As the drain bias is increased and the reduced transmission region (around $-0.2 \mathrm{eV}$ ) falls within the $E f_{S}-E f_{D}$ window, the drive current of the defected devices reduces. At even higher biases, the transmission, as well as the conductance of the channel (slope of the $I_{D^{-}} V_{D}$ ) is rectified. Due to this dynamic dependence of the current on the drain bias, a single value for the channel resistance cannot be easily defined as in the case of the $(12,0)$ CNT. Physically this difference between the performances of the two different diameter defected devices can be explained by the fact that in the larger diameter CNT, the electrons have larger phase space and more paths to avoid scattering off the vacancy, making its effect less important.

The software develped for this research study is now available on the nanoHUB for free usage by the community at large. Readers of the article can verify the findings of this work with this online tool and study a variety of other device configurations. We note that at high bias, incorporation of several defects, still has potential convergence issues due to the large internal charge oscillations, as mentioned in the previous paragraph.

In this letter, we examine the role of atomistic vacancy defects in altering metallic nanotube transport characteristics from the ballistic limit. A single vacancy in a CNT channel can severely alter the potential energy and charge distribution along the channel of the device. In small diameter CNTs, a single vacancy defect can reduce the drive current capabilities of the CNT by half. Multiple defects can decrease the performance even more. The reduction, however, is much less in larger diameter CNTs. Design of devices using these quasi-one-dimensional channels for certain applications should take into consideration the effect that a single defect can degrade the current of the device significantly.

This work was supported by the Semiconductor Research Corporation (SRC). Computational resources on nanohub.org have been used to conduct the simulations. One of the authors (N.N.) would like to thank Gengchiau Liang for helpful discussions.

${ }^{1}$ P. L. McEuen, M. S. Fuhrer, and H. Park, IEEE Trans. Nanotechnol. 1, 78 (2002).

${ }^{2}$ A. Javey, R. Tu, D. Farmer, J. Guo, R. Gordon, and H. Dai, Nano Lett. 5, 345 (2005).

${ }^{3}$ J. Appenzeller, Y. M. Lin, J. Knoch, Z. Chen, and P. Avouris, IEEE Trans. Electron Devices 52, 12 (2005).

${ }^{4}$ J. Chen, C. Klinke, A. Afzali, and Ph. Avouris, Appl. Phys. Lett. 86, 123108 (2005).

${ }^{5}$ F. Kreup, A. P. Graham, M. Liebau, G. S. Duesberg, R. Seidel, and E. Unger, Tech. Dig. - Int. Electron Devices Meet. 2004, 683.

${ }^{6}$ Z. Yao, C. Kane, and C. Dekker, Phys. Rev. Lett. 84, 2941 (2000).

${ }^{7}$ A. Patil, T. Ohashi, A. Buldum, and L. Dai, Appl. Phys. Lett. 89, 103103 (2006).

${ }^{8}$ A. A. El-Barbary, R. H. Telling, C. P. Ewels, M. I. Heggie, and P. R. Briddon, Phys. Rev. B 68, 144107 (2003).

${ }^{9}$ A. Hashimoto, K. Suenaga, A. Gloter, K. Urita, and S. Ijima, Nature (London) 430, 870 (2004).

${ }^{10}$ Y. Fan, B. R. Goldsmith, and P. G. Collins, Nat. Mater. 4, 906 (2005).

${ }^{11}$ A. V. Krasheninnikov, K. Nordlund, M. Sirvio, E. Salonen, and J. Keinonen, Phys. Rev. B 63, 245405 (2001).

${ }^{12}$ A. V. Krasheninnikov, K. Nordlund, and J. Keinonen, Phys. Rev. B 65, 165423 (2002).

${ }^{13}$ C. Gomez-Navarro, P. J. De Pablo, J. Gomez-Herrero, B. Biel, F. J. Garcia-Vidal, A. Rubio, and F. Flores, Nat. Mater. 4, 534 (2005).

${ }^{14}$ N. Neophytou, D. Kienle, E. Polizzi, and M. P. Anantram, Appl. Phys. Lett. 88, 242106 (2006).

${ }^{15}$ J. Guo, S. Datta, M. Lundstrom, and M. P. Anantram, Int. J. Multiscale Comp. Eng. 2, 257 (2004).

${ }^{16} \mathrm{http} / / / \mathrm{www}$. nanohub.org/tools/cntfet/

${ }^{17}$ M. P. Anantram and T. R. Govindan, Phys. Rev. B 58, 4882 (1998).

${ }^{18}$ L. Chico, L. Benedict, S. Louie, and M. Cohen, Phys. Rev. B 54, 2600 (1996). 